\title{
ATLAS searches for resonances decaying to boson pairs
}

\author{
Andrey Ryzhov ${ }^{1, \star}$, on behalf of the ATLAS Collaboration \\ ${ }^{1}$ Institute for High Energy Physics of National Research Centre "Kurchatov Institute", Protvino, Russia
}

\begin{abstract}
Many extensions to the Standard Model predict new particles decaying into two bosons ( $W, Z$, photon, or Higgs bosons) making these important signatures in the search for new physics. Searches for such diboson resonances have been performed in final states with different numbers of leptons, photons, jets and $b$-jets where new jet substructure techniques to disentangle the hadronic decay products in highly boosted configuration are being used. This document summarizes recent ATLAS searches for resonances decaying to diboson final states, $V V, V H$ and $H H$ with LHC Run 2 data collected.
\end{abstract}

\section{Introduction}

The discovery of the Higgs boson [1] with a mass of approximately $125 \mathrm{GeV}$ in 2012 finalizes the Standard Model (SM) in the description of particle interactions at energies up to a few hundred GeV. Despite the fact that a lot of precision measurements and searches with data from the Large Hadron Collider (LHC) show no significant deviations from the SM, some unanswered questions still exist. Therefore one of the main purposes of particle physics research at the LHC is to seek new intimations of physics Beyond the Standard Model (BSM). Many of BSM models, motivated by hierarchy and naturalness arguments [2], predict the existence of new heavy resonances decaying into diboson, such as composite Higgs models [3], warped extra dimensions [4], models with an extended Higgs sector [5] and Grand Unified Theories [6].

In order to evaluate the sensitivity, to optimise the event selections, and for comparison with the data, a number of models are used as benchmarks. Limits on cross-sections as a function of the particle mass are obtained in the framework of three representative benchmark models: an extended Higgs sector serves as benchmark model for spin-0 resonances, Heavy Vector Triplets (HVT) Model A (which has comparable branching ratios to fermions and gauge bosons) and Model B (which has enhanced couplings to gauge bosons and where the fermionic couplings are suppressed) for spin-1 in form of $W^{\prime}$ and $Z^{\prime}$ [7], and bulk Randall-Sundrum Gravitons (RSG) [8] for spin-2 resonances.

In this document, the results from recently performed searches for resonant production of $V V, V H$ and $H H$ are summarised. They are carried out using data taken with the ATLAS detector [9] during the 2015-2016 run of the LHC (Run 2).

The searches are performed by looking for localized excesses above the smoothly falling SM background. For this purpose, the mass of the diboson system is used as the final discriminant. This is the invariant mass unless $Z$ boson decays to neutrinos $(v)$ are included, where only the transverse mass reconstruction is feasible.

^e-mail: Andrey.Ryzhov@ihep.ru 


\section{Experimental techniques}

All models predict the existence of new particles with masses approximately few $\mathrm{TeV}$, hence candidate decay products (especially $W, Z$ or $H$ bosons reconstructed through hadronic decays) must have high transverse momenta. In this case the distance in $\eta-\varphi$ space between direction of two quarks emerging from the boson decay can be approximated by $\Delta R=\sqrt{\Delta \eta^{2}+\Delta \phi^{2}} \approx 2 m / p_{T}$, where $m$ and $p_{T}$ are the mass and the transverse momenta of the parent boson $(W, Z, H)$, respectively. When the resonance mass is significantly higher than the boson mass, the $q q$ pair from the boson decay can be collimated. And hadrons from the two quarks overlap in the detector and are more efficiently reconstructed as a single large-radius jet (large- $R$ jet, or $J$ ). Therefore two different reconstruction techniques for the hadronic decay are considered: resolved and merged. The resolved reconstruction attempts to identify boson decay products as two separate small-radius anti- $k_{t}$ jets with a radius parameter of 0.4 (small$R$ jet, or $j$ ), while the merged reconstruction uses jet substructure techniques to identify the boson hadronic decay reconstructed as a large- $R$ jet using the anti- $k_{t}$ algorithm with $R=1$. In order to remove a large contamination by soft radiation (pile-up, underlying event) a trimming algorithm [10] is applied to the large- $R$ jets, retaining only components (anti- $k_{t}$ jets with $R=0.2$ ) carrying at least $5 \%$ of the original jet's energy. The large- $R$ jet mass is calculated from combining calorimeter and tracking information, to improve jet mass resolution [11].

Vector boson tagging technique [12] using a variable named $D_{2}$ is applied to the large- $R$ jets to select them from the $V \rightarrow q q$ decays. This $D_{2}$ variable is optimised to distinguish between jets originating from a single parton and those coming from the two-body decay of a heavy particle. The $V$ boson jets are then selected by requiring that the large- $R$ jet mass $m_{J}$ should be in a $p_{T}$-dependent window centred around the expected value of the boson mass from simulations. Higgs bosons decaying to bottom quarks are identified by applying a dedicated Higgs-tagging technique [13] to the large- $R$ jets. This algorithm proceeds by ghost-associating [14] anti- $k_{t}$ jets $(R=0.2)$ reconstructed from tracks in the Inner Detector (ID) and applying a $b$-tagging algorithm [15]. The operating point of the $b$-tagging algorithm is chosen such that the $b$-tagging efficiency for jets containing $b$-hadrons is $77 \%$. In addition, a mass window cut around the SM Higgs mass of $125 \mathrm{GeV}$ is applied to the large- $R$ jet.

\section{Analyses overview and results}

This section summarizes the individual searches presented in this article. Subsection 3.1 gives the results for the $\mathrm{VV}, \mathrm{VH}, \mathrm{HH}$ searches in fully hadronic decay modes, followed by the results for $V V$ resonances in semileptonic decay modes in Subsect. 3.2. And finally a short summary is given in Table 1, which shows observed excluded resonance masses at the $95 \%$ confidence level (CL) for all analyses. The full description of each analysis can be found in the reference papers. These analyses are performed using data collected with the ATLAS detector at $\sqrt{s}=13 \mathrm{TeV}$ with an integrated luminosity that differs for each particular analysis and that will be indicated in each subsection.

\section{1 $V V, V H$ and $H H$ resonances in fully hadronic decay modes}

The $\boldsymbol{V} \boldsymbol{V} \rightarrow \boldsymbol{q q q \boldsymbol { q }}$ analysis [16] is performed only in the merged regime using $36.7 \mathrm{fb}^{-1}$ of protonproton collision data at a centre-of-mass energy of $\sqrt{s}=13 \mathrm{TeV}$ recorded with the ATLAS detector at the Large Hadron Collider in 2015 and 2016. Events are required to contain at least two large- $R$ jets with $|\eta|<2.0$ (to guarantee a good overlap with the tracking acceptance) and mass $m_{J}>50 \mathrm{GeV}$. The leading (highest $p_{T}$ ) large- $R$ jet must have $p_{T}>450 \mathrm{GeV}$ and the subleading (second highest $p_{T}$ ) large- $R$ jet must have $p_{T}>200 \mathrm{GeV}$. The invariant mass of the dijet system formed by these two jets 
must be $m_{J J}>1.1 \mathrm{TeV}$ to avoid inefficiencies due to the minimum jet- $p_{T}$ requirements. Boson-jet candidates are identified by applying a boson-tagging procedure. Further requirements are applied on the number of tracks in the two jets, their rapidity difference and their $p_{T}$ asymmetry. This selection strongly suppresses the large backgrounds due to SM multi-jet events. Other contributions to the background are small. The dijet invariant-mass spectrum of the background is estimated directly from data using a parametric form, and the search proceeds by seeking resonant structures in the falling spectrum of the background. The ability of the parametric shape to model distributions is tested with validation regions from collision data (see Figure 1(left)). No significant excess over the background is found in the signal regions, as shown for the WZ selection in Figure 1(right) and thus 95\% CL exclusion limits are set on the production cross section times branching fraction in the HVT and bulk RS models as shown in Figure 2.
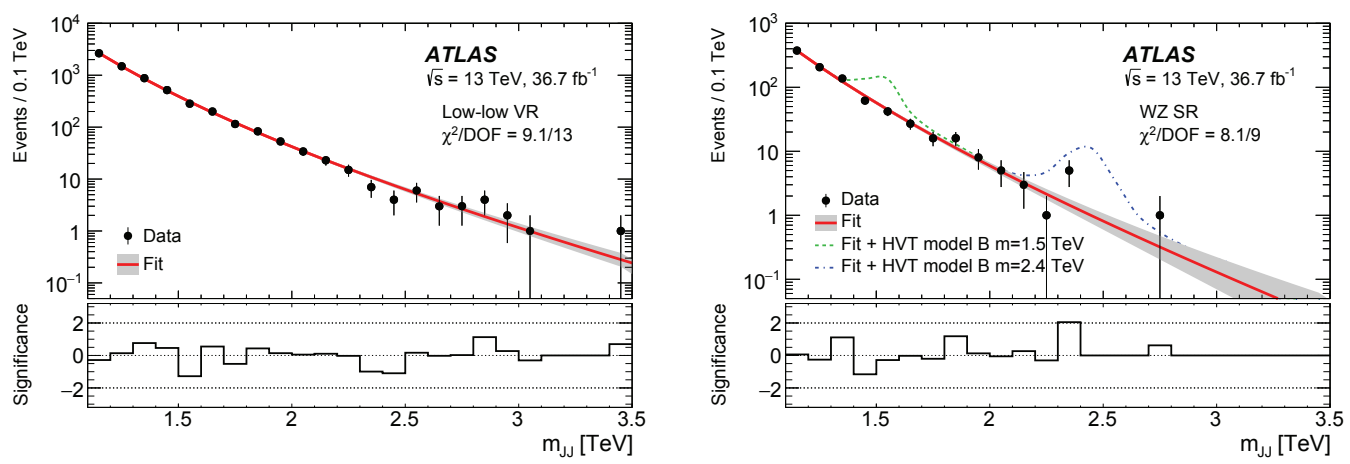

Figure 1. Left: Dijet mass distributions for data in the sideband validation regions. The shaded bands represent the uncertainty in the background expectation due to the maximum-likelihood fit's statistical uncertainty. The lower panels show the significance of the observed event yield relative to the background fits. Right: Dijet mass distributions for data in the $W Z$ signal region. The red lines correspond to the result of the fit and the shaded bands represent the uncertainty in the background expectation. Expected signals are shown for the HVT model B and the bulk RS model. The predictions for $G_{K K}$ production are multiplied by a factor of 10 . Both figures are taken from ref. [16]

The $\boldsymbol{V} \boldsymbol{H} \rightarrow \boldsymbol{q q} \boldsymbol{\boldsymbol { b }} \overline{\boldsymbol{b}}$ analysis [17] is carried out with $36.1 \mathrm{fb}^{-1}$ of Run 2 data. There is only merged regime which uses two large- $R$ jets selection with $p_{T}>450$ (250) $\mathrm{GeV}$ for the leading (sub-leading) jet, $|\eta|<2$ and an invariant mass larger than $50 \mathrm{GeV}$. The analysis is performed categorizing the signal in four regions, namely $W H 1 b$-tag, $W H 2$-tag, $Z H 1 b$-tag and $Z H 2 b$-tag. Signal and backgrounds from $t \bar{t}$ and $W / Z+$ jets production are modelled with Monte Carlo (MC) simulation. While multijet MC events are used as a cross-check, the primary multijet background estimation is performed using data. This background shape is extracted from $0 b$-tag "SR", which consists of $\approx 99 \%$ multijet events. The normalization of each distribution is obtained using the sideband regions. The data are in agreement with the Standard Model expectations (see Figure 3(left)), with the largest excess observed at $m_{J J} \approx$ $3.0 \mathrm{TeV}$ in the $Z H$ channel with a local significance of $3.3 \sigma$. The global significance of this excess is $2.1 \sigma$ (see Figure 3(right)).

The search for $\boldsymbol{H} \boldsymbol{H} \rightarrow \boldsymbol{b} \overline{\boldsymbol{b}} \boldsymbol{b} \overline{\boldsymbol{b}}$ final state [18] is done in both boosted and resolved regimes, covering high and low energy regimes, respectively. The results presented here benefit from an integrated luminosity of $13.3 \mathrm{fb}^{-1}$. The merged analysis selects events with at least two anti- $k_{t} R=1.0$ jets 

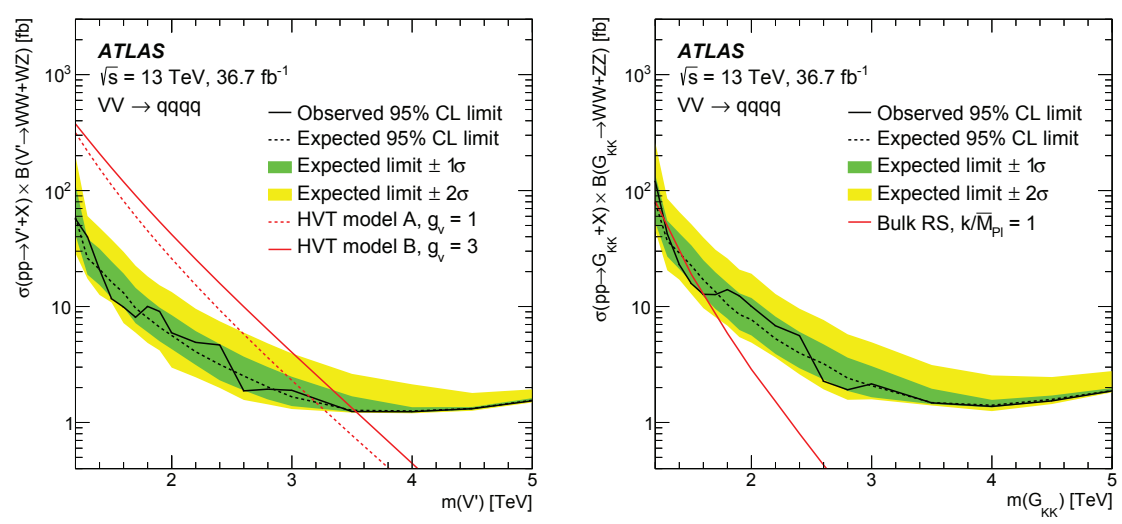

Figure 2. Upper limits at the $95 \% \mathrm{CL}$ on the cross section times branching ratio for (left) $W W+W Z$ production as a function of $V^{\prime}$ mass, (right) $W W+Z Z$ production as a function of $G_{K K}$ mass. The dotted and solid red lines show the predicted cross section times branching ratio as a function of resonance mass for the HVT Models A and B with $g_{V}=1$ and $g_{V}=3$, respectively, or the bulk RS model with $k / \bar{M}_{P l}=1$. Both figures are taken from ref. [16]
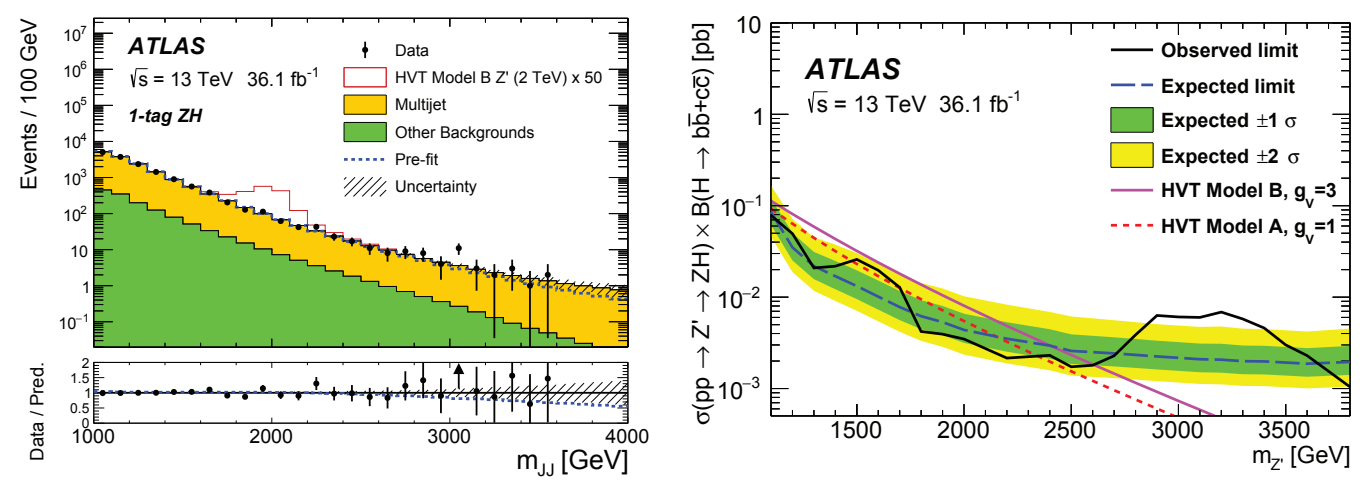

Figure 3. Left: The $m_{J J}$ distributions in the $V H$ signal regions for data (points) and background estimate (histograms) after the likelihood fit for events in the 1-tag categories. The pre-fit background expectation is given by the blue dashed line. The expected signal distributions (multiplied by 50) for a HVT benchmark Model B $V^{\prime}$ boson with $2 \mathrm{TeV}$ mass are also shown. Right: The observed and expected cross-section upper limits at the 95\% confidence level on the cross section times branching ratio for HVT Model A and Model B in the $\mathrm{ZH}$ signal regions. The red and magenta curves show the predicted cross-sections as a function of resonance mass for the models considered. Both figures are taken from ref. [17]

with $p_{T}>450$ (250) GeV for the leading (sub-leading) jet, $|\eta|<2$ and an invariant mass larger than $50 \mathrm{GeV}$. Since high-mass resonances tend to produce jets that are more central than multijet background processes, the two large- $R$ jets are required to have a separation $|\Delta \eta|<1.7$. The resolved selection begins with the requirement that the event contains at least four $b$-tagged anti- $k_{t} R=0.4$ jets with $p_{T}>30 \mathrm{GeV}$ and $|\eta|<2.5$. The leading jets are combined in pairs forming the Higgs candidates and requiring to pass $\Delta R$ and $p_{T}$ cuts which depend on invariant mass of $H H$ system. For both the 
boosted and the resolved analyses the dominant background is QCD multijet which is estimated in a side band region and validated in a control region in the $m_{H_{1}}, m_{H_{2}}$ plane as shown in Figure 4(left), where $m_{H}$ refers to the invariant mass of the Higgs candidate. Good agreement is observed between the data and the background prediction (see Figure 4(right)).
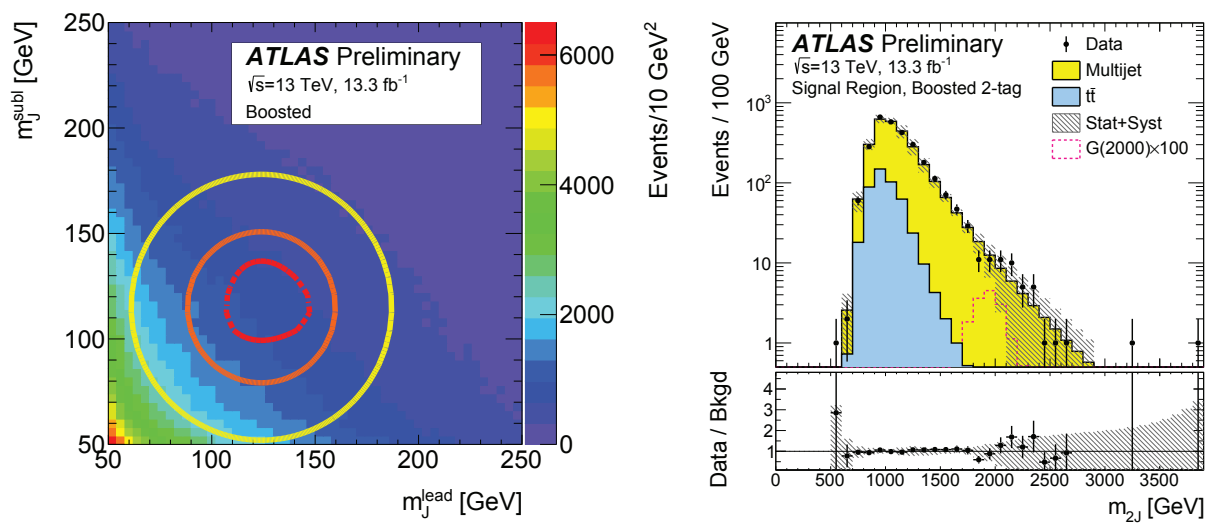

Figure 4. Left: The $m_{J}^{\text {subl }}$ vs. $m_{J}^{\text {lead }}$ distribution for the boosted analysis background model. The signal region is the area surrounded by the dashed red contour line, centred on $\left(m_{J}^{\text {lead }}=124 \mathrm{GeV}, m_{J}^{\text {subl }}=115 \mathrm{GeV}\right)$. The control region is the area between the signal region and the orange contour line. The sideband region is the area between the control region and the yellow contour line. Right: Distributions of $m_{2 J}$ in the signal regions of the boosted analysis for the two-tag-split sample compared to the predicted backgrounds. The grey hatched bands represent the combined statistical and systematic uncertainties in the total background estimates. The expected signal shape for a $G_{K K}$ resonance of mass $2.0 \mathrm{TeV}$ is also shown. Both figures are taken from ref. [18]

\section{2 $V V$ resonances in semileptonic decay modes}

All semileptonic analysis is carried out with $36.1 \mathrm{fb}^{-1}$ of LHC proton-proton collision data collected at $\sqrt{s}=13 \mathrm{TeV}$ with the ATLAS detector in 2015 and 2016. One boson candidate is required to be formed by a $l v, l l$ or $v v$ pair and the other boson by a $q q$ pair or single jet, where both bosons should satisfy $V$ mass window cuts. Events with two additional small- $R$ jets (referred to as tag-jets) with large pseudorapidity separation and invariant mass are classified as vector-boson fusion (VBF) candidates, while absence of this topology is interpreted as gluon-gluon fusion/Drell-Yan (ggF/DY) production.

The $\boldsymbol{Z V} \rightarrow \boldsymbol{l l} \boldsymbol{q} \boldsymbol{q}$ search [19] explores the VBF and $\mathrm{ggF}$ production of a heavy Higgs boson $H$, the VBF and DY production of an HVT $W^{\prime}$ boson, and the ggF production of a bulk RS graviton $G_{K K}$. It also utilises both the merged and resolved reconstruction for the $V \rightarrow q q$ decay. The search begins with the identification of the $Z \rightarrow l l$ decay (the leading lepton must satisfy $E_{T}\left(p_{T}\right)>28 \mathrm{GeV}$ and the dilepton invariant mass $m_{l l}$ is required to be consistent with the $\mathrm{Z}$ boson mass), followed by classifying events into the $\operatorname{VBF}\left(\eta_{t a g_{1}} \cdot \eta_{t a g_{2}}<0,\left|\Delta \eta_{t a g}\right|>4.7, m_{t a g}>770 \mathrm{GeV}\right)$ or $\mathrm{ggF} / \mathrm{DY}$ categories and finally the selection of either the $Z V \rightarrow l l J$ or $Z V \rightarrow l l j j$ final states. Merged events are further required to have $p_{T}^{J}>200 \mathrm{GeV}$ and $\min \left(p_{l l}, p_{J}\right) / m_{l l J}>0.3$ for the $H \rightarrow Z Z$ search and $>0.35$ for the $W^{\prime} \rightarrow Z W$ and $G_{K K} \rightarrow Z Z$ searches. Two signal regions are defined, high-purity (HP) and low-purity (LP) SR, that depend on boson tagging working point requirement. Events that have failed merged selection are required to have at least two small- $R$ jets with $p_{T}>60 \mathrm{GeV}$. The kinematic quantity $\sqrt{\left(p_{T}^{l l}\right)^{2}+\left(p_{T}^{j j}\right)^{2}} / m_{l l j j}$ is required to be greater than 0.4 for $H \rightarrow Z Z$ and 0.5 for $W^{\prime} \rightarrow Z W$ 
and $G_{K K} \rightarrow Z Z$. The dijet invariant mass must be in the window [70,105] GeV for $Z \rightarrow q q$ and in the window $[62,97] \mathrm{GeV}$ for $W \rightarrow q q$. Also the $Z V \rightarrow l l j j$ candidates are divided into two signal regions: events with two $b$-tagged jets and events with fewer than two $b$-tagged jets. Multiple signal regions are defined to enhance the sensitivity of the search. The dominant backgrounds are the $Z+$ jets, top quark and diboson processes. Their contributions are estimated from a combination of MC and data-driven techniques. The shapes of kinematic variables are taken from MC simulations. In order to extract the signal rate information, a simultaneous maximum-likelihood fit is performed to the observed distributions of the final discriminants in the signal regions, $m_{l l J}$ or $m_{l l j j}$. The data are found to be consistent with the SM background predictions and no evidence of heavy resonance production is observed (see Figure 5). Limits are presented in Figure 6.
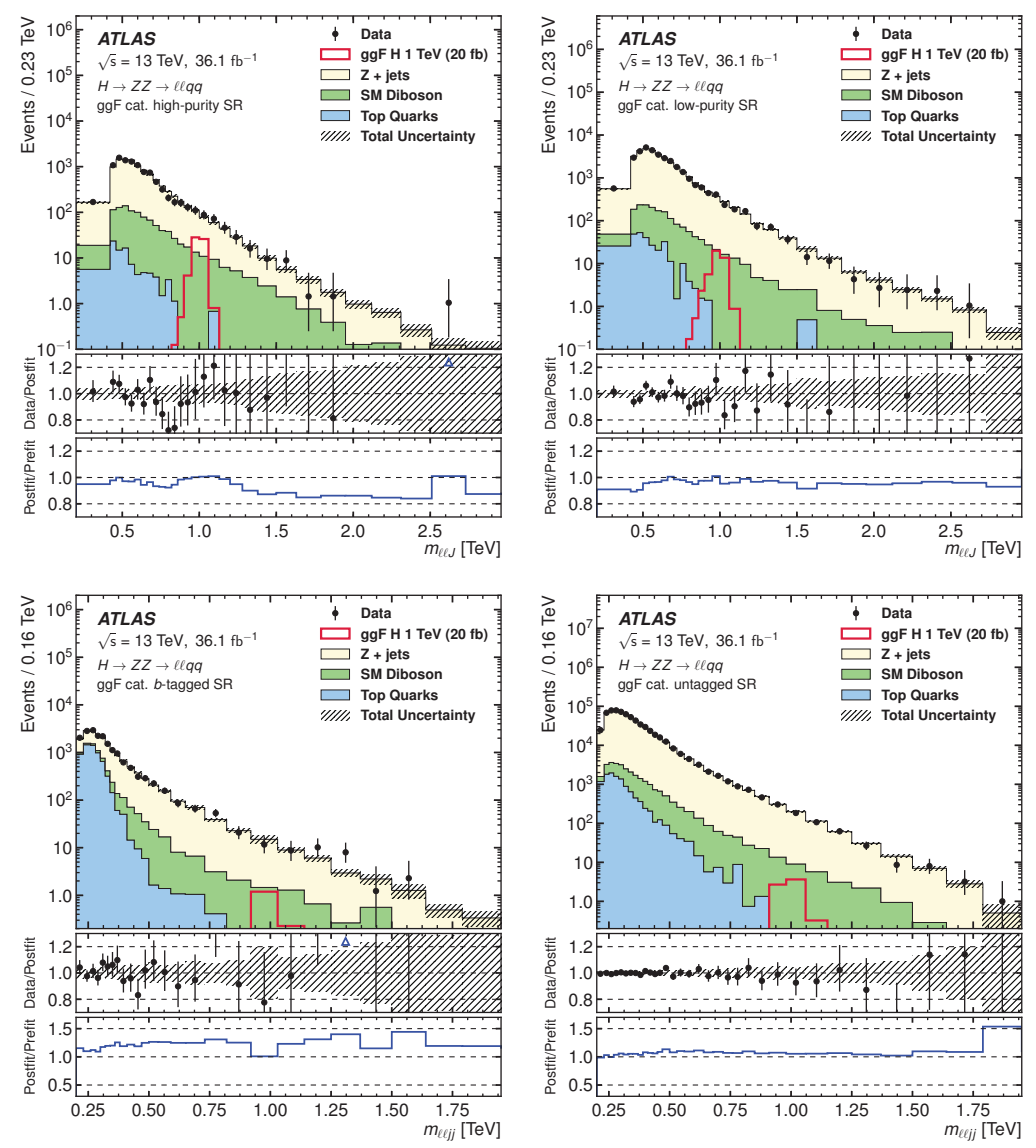

Figure 5. Comparisons of the observed data and expected background distributions of the final discriminants of the ggF category for the $H \rightarrow Z Z \rightarrow l l q q$ search: $m_{l l J}$ of (top left) high-purity and (top right) low-purity signal regions; $m_{l l j j}$ of (bottom left) $b$-tagged and (bottom right) untagged signal regions. For illustration, expected distributions from the ggF production of a $1 \mathrm{TeV}$ Higgs boson with $\sigma \times B(H \rightarrow Z Z)=20 \mathrm{fb}$ are also shown. The middle panes show the ratios of the observed data to the background predictions. The uncertainty in the total background prediction, shown as bands, combines statistical and systematic contributions. The bottom panes show the ratios of the post-fit and pre-fit background predictions. All figures are taken from ref. [19] 

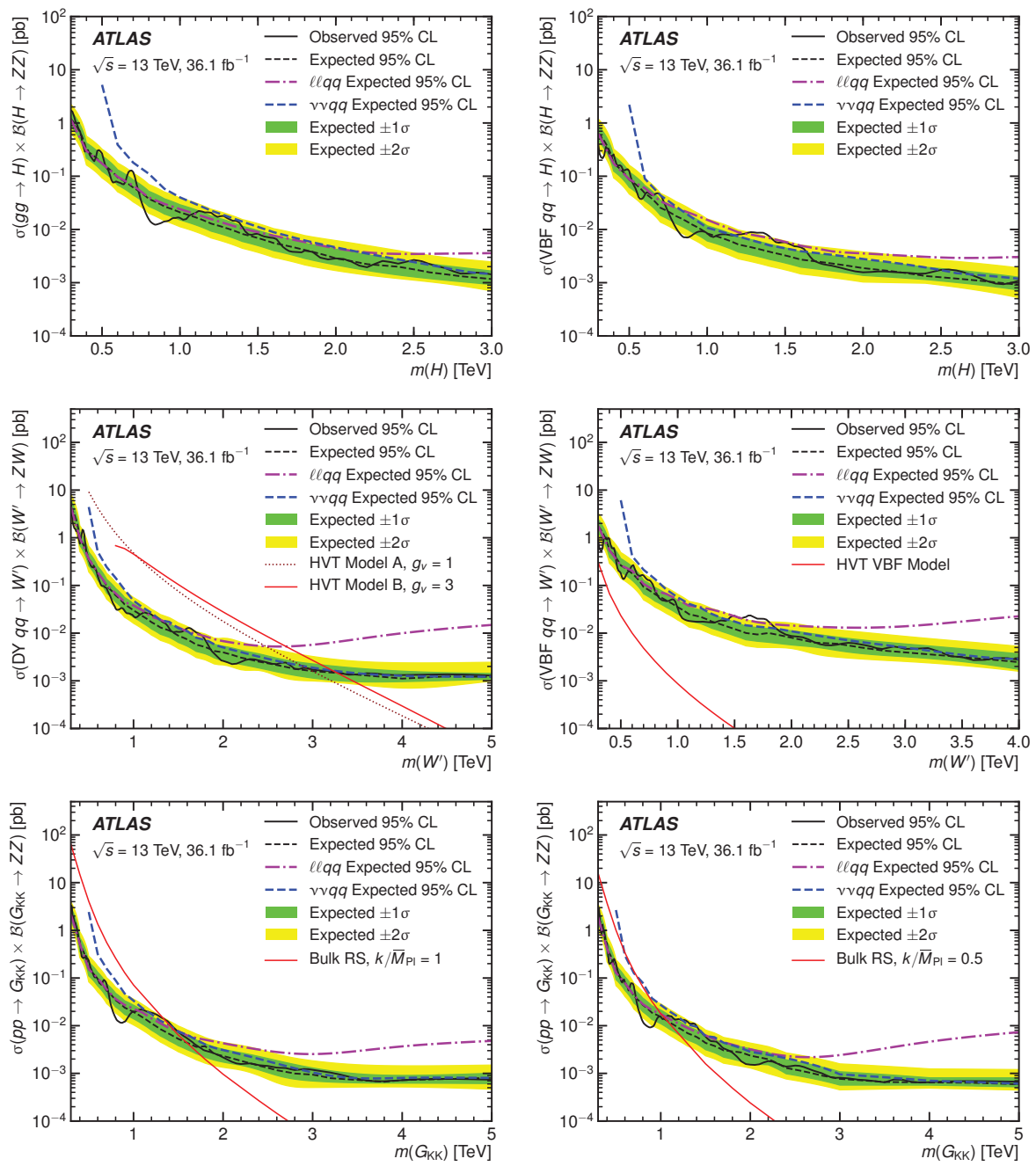

Figure 6. Observed (black solid curve) and expected (black dashed curve) 95\% CL upper limits: on $\sigma \times B(H \rightarrow$ $Z Z$ ) for the (top left) $g g F$ and (top right) VBF production of a heavy Higgs boson as a function of its mass; on $\sigma \times B\left(W^{\prime} \rightarrow Z W\right)$ for the (middle left) DY and (middle right) VBF production of a $W^{\prime}$ boson in the HVT model as a function of its mass; on $\sigma \times B\left(G_{K K} \rightarrow Z Z\right)$ for the production of a $G_{K K}$ in the bulk RS model with couplings of (bottom left) $k / \bar{M}_{P l}=1$ and (bottom right) $k / \bar{M}_{P l}=0.5$ as a function of the graviton mass, combining $l l q q$ and $v v q q$ searches. Limits expected from individual searches (dashed curves in blue and magenta) are also shown for comparison. Limits are calculated in the asymptotic approximation below $2 \mathrm{TeV}$ and are obtained from pseudo-experiments above that. All figures taken from ref. [19]

The $Z \boldsymbol{V} \rightarrow \boldsymbol{v} \boldsymbol{v q q}$ selection [19] closely follows the $V Z \rightarrow l l q q$ search with additional requirements to remove multijet backgrounds but without the resolved selection. An initial selection is made by requiring $E_{\text {miss }}>250 \mathrm{GeV}$, and vetoing events with electrons or muons. The multijet background, originating primarily from the presence of mismeasured jets, and non-collision backgrounds 
are suppressed by using a track-based missing transverse momentum, $\vec{p}_{T}^{\text {miss }}$. Due to the presence of neutrinos in the final state, it is not possible to fully reconstruct the invariant mass of the $v v J$ system, so the transverse mass is used as the final discriminant: $m_{T}=\sqrt{\left(E_{T, J}+E_{T}^{\text {miss }}\right)^{2}+\left(\vec{p}_{T, J}+{\overrightarrow{E_{T}}}_{\text {miss }}\right)^{2}}$, where $E_{T, J}=\sqrt{m_{J}^{2}+p_{T, J}^{2}}$. The dominant backgrounds are $Z+$ jets, $W+$ jets and $\bar{t} \bar{t}$ processes. Data control regions are defined to check the modelling of each contribution. As in the llqq search, the shapes of kinematic variables, including the final discriminant $m_{T}$, are taken from MC simulations. Figure 7 shows that no significant excess over the background is found in the signal regions. Limits are presented in Figure 6.
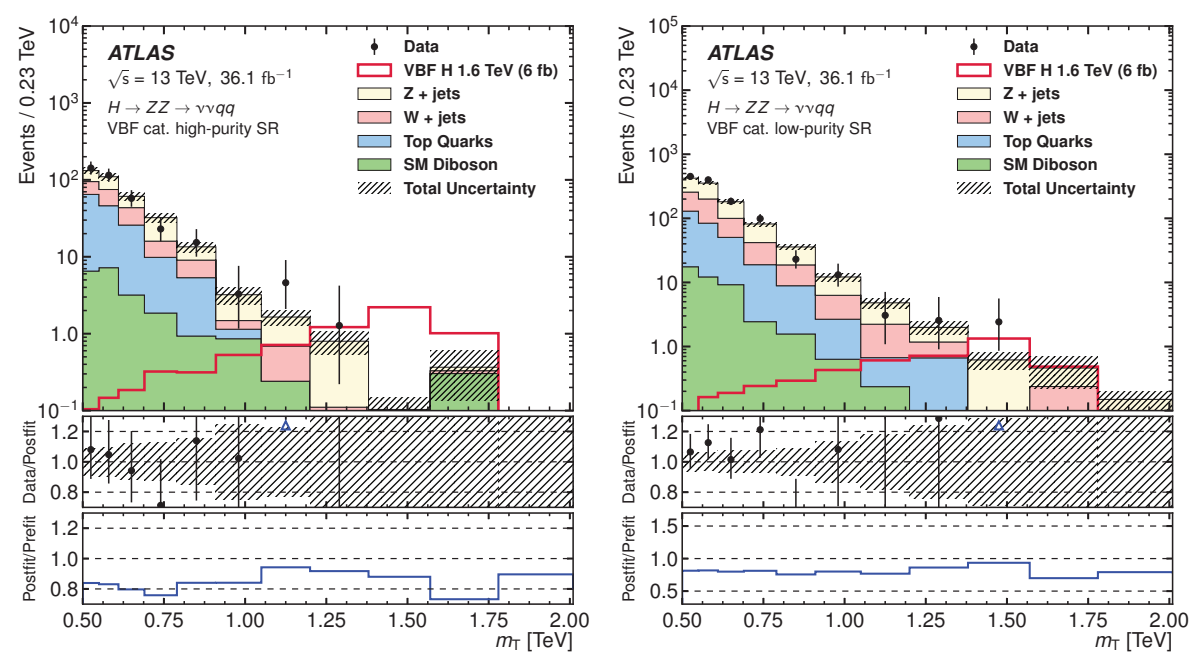

Figure 7. Comparisons of the observed data and expected background distributions of $m_{T}$ in the VBF category of the $H \rightarrow Z Z \rightarrow v v q q$ search: (left) high-purity and (right) low-purity signal regions. For illustration, expected distributions from the VBF production of a $1.6 \mathrm{TeV}$ Higgs boson with $\sigma \times B(H \rightarrow Z Z)=6 \mathrm{fb}$ are also shown. The middle panes show the ratios of the observed data to the background predictions. The bottom panes show the ratios of the post-fit and pre-fit background predictions. Both figures taken from ref. [19]

The $\boldsymbol{W} \boldsymbol{V} \rightarrow \boldsymbol{l v q} \boldsymbol{q}$ analysis [20] is very similar to the previous $Z V \rightarrow l l q q$, also with resolved and merged regime, but some additional requirements are used to eliminate the multijet contamination $\left(E_{T}^{\text {miss }}>100 \mathrm{GeV}, p_{T}(l v)>200 \mathrm{GeV}, E_{T}^{\text {miss }} / p_{T}(l v)>0.2\right)$. The analysis selects events that contain exactly one charged signal lepton and no additional veto electrons or muons, then events are categorized to VBF or DY categories and are assigned to merged and resolved regions. Studies using simulated events show that $W+$ jets and $t \bar{t}$ production are the dominant background sources. The shapes of the mass distributions for events from SM production of $W+$ jets and $t \bar{t}$ are modeled using simulated events. The results are extracted by performing a simultaneous binned maximum-likelihood fit to the $m(W V)$ distributions in the signal regions and the control regions for $W+$ jets and $t \bar{t}$. The $m(W V)$ distributions are presented in Figure 8. The data are compatible with the Standard Model background hypothesis and no significant excess has been found. 

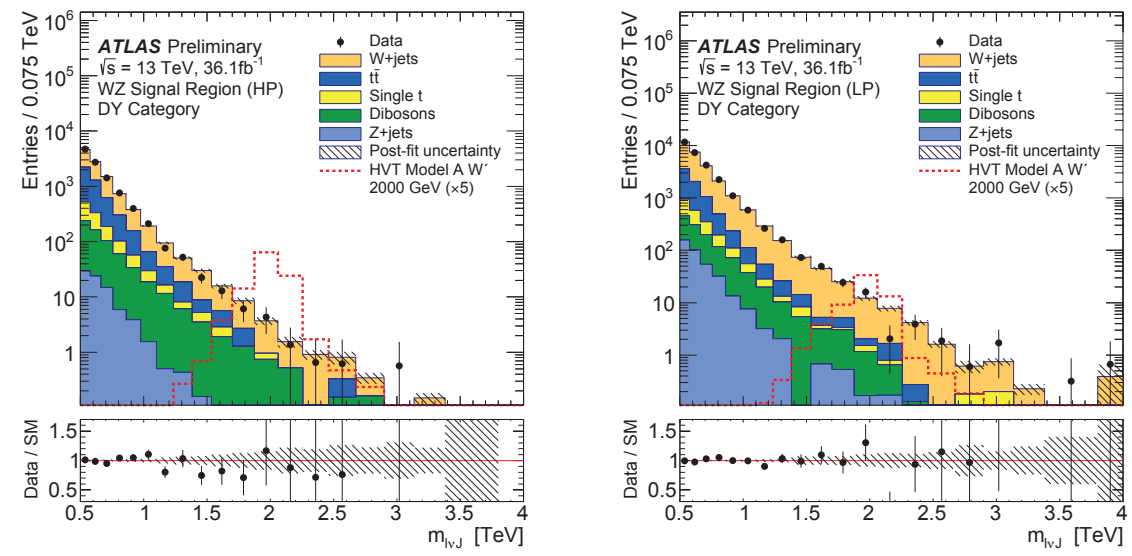

Figure 8. Post-fit signal region $m(W V)$ distributions in the DY category. Event failing the VBF selection are assigned to this category. The merged high-purity (HP) sample of WZ (left) events, the merged low-purity (LP) sample of $W Z$ (right) events are presented. The background expectation is shown after the profile likelihood fit to the data and signal expectations are overlaid. The HVT Model A signal at $2000 \mathrm{GeV}$ is presented. The band denotes the statistical and systematic uncertainty on the background after the fit to the data. The lower panels show the ratio of the observed data to the SM background estimation. In all regions, the overflow events are included in the last bin. Both figures are taken from ref. [20]

Table 1. Observed excluded resonance masses (at 95\% CL) for the HVT and bulk RS models.

\begin{tabular}{|c||c|c|c|}
\hline \multirow{2}{*}{ Analysis } & \multicolumn{3}{|c|}{ Excluded mass range $[\mathrm{TeV}]$} \\
\cline { 2 - 4 } & $\begin{array}{c}\text { HVT } W^{\prime} \text { Model A } \\
(\text { Model B) }\end{array}$ & $\begin{array}{c}\text { HVT Z Model A } \\
\text { (Model B) }\end{array}$ & $\begin{array}{c}\mathrm{RS} \mathrm{G}_{K K}, k / \bar{M}_{P l}=1 \\
\left(k / \bar{M}_{P l}=0.5\right)\end{array}$ \\
\hline$V V \rightarrow q q q q$ & $1.1-3.0(1.2-3.3)$ & $1.2-2.2(1.2-2.8)$ & $1.3-1.45$ \\
\hline$V V \rightarrow q q \bar{b} \bar{b}$ & $1.1-2.4(1.1-2.5)$ & $\begin{array}{c}1.1-1.48,1.7-2.35 \\
(1.1-2.6)\end{array}$ & none \\
\hline$H H \rightarrow b \bar{b} b \bar{b}$ & none & none & $0.36-0.86$ \\
\hline$Z V \rightarrow l l q q, v v q q$ & $<2.9(<3.2)$ & none & $<1.3(<1.0)$ \\
\hline$W V \rightarrow l v q q$ & $<2.82(<2.98)$ & $<2.75(<3.09)$ & $<1.76$ \\
\hline
\end{tabular}

\section{Conclusion}

A brief summary of the most recent searches for resonances decaying to diboson final states, $V V$, $\mathrm{VH}$ and $\mathrm{HH}$ using ATLAS data from the 2015 and 2016 run at the LHC have been presented. No discrepancies with respect to the Standard Model expectations are observed and thus 95\% CL exclusion limits are set on the production cross section times branching ratios in a number of benchmark scenarios. Observed excluded resonance masses at the 95\% confidence level for the HVT and bulk RS models are shown in the table 1 . The utilization of unprecedented luminosity of $\approx 85 \mathrm{fb}^{-1}$ available at the end of the whole LHC Run 2 should give significant improvements on the exclusion limits. 


\section{References}

[1] ATLAS Collaboration, Observation of a new particle in the search for the Standard Model Higgs boson with the ATLAS detector at the LHC, Phys. Lett. B 716 (2012) p. 1, arXiv: 1207.7214 [hepex]

[2] N. Arkani-Hamed, S. Dimopoulos and G. R. Dvali, The Hierarchy problem and new dimensions at a millimeter, Phys. Lett. B 429 (1998) 263, arXiv: hep-ph/9803315.

[3] M. J. Dugan, H. Georgi and D. B. Kaplan, Anatomy of a Composite Higgs Model, Nucl. Phys. B 254 (1985) 299.

[4] L. Randall and R. Sundrum, A Large mass hierarchy from a small extra dimension, Phys. Rev. Lett. 83 (1999) 3370, arXiv: 9905221.

[5] D. de Florian et al., Handbook of LHC Higgs Cross Sections: 4. Deciphering the Nature of the Higgs Sector, (2016), arXiv: 1610.07922.

[6] J. C. Pati and A. Salam, Lepton Number as the Fourth Color, Phys. Rev. D 10 (1974) 275.

[7] D. Pappadopulo, A. Thamm, R. Torre, A. Wulzer, Heavy vector triplets: bridging theory and data, JHEP 9 (2014) 60, arXiv:1402.4431 [hep-ph].

[8] K. Agashe, H. Davoudiasl, G. Perez, A. Soni, Warped gravitons at the CERN LHC and beyond, Phys. rev. D76 (2007) 036006, arXiv:hep-ph/0701186.

[9] ATLAS Collaboration, The ATLAS Experiments at the CERN Large Hadron Collider, JINST 3 (2008) S08003.

[10] D. Krohn, J. Thalr, L.-T. Wang, Jet trimming, JHEP 2 (2010) 84, arXiv:0912.1342 [hep-ph].

[11] ATLAS Collaboration, ATLAS Combined Mass resolution and W mass reconstruction performance, JETM-2017-002, https://atlas.web.cern.ch/Atlas/GROUPS/PHYSICS/PLOTS/JETM2017-002.

[12] ATLAS Collaboration, Identification of Boosted, Hadronically-Decaying $W$ and $Z$ Bosons in $\sqrt{s}=13 \mathrm{TeV}$ Monte Carlo Simulations for ATLAS, ATL-PHYS-PUB-2015-033, https://cds.cern.ch/record/2041461.

[13] ATLAS Collaboration, Expected Performance of Boosted Higgs $(\rightarrow \quad b \bar{b})$ Boson Identification with the ATLAS Detector at $\sqrt{s}=13 \mathrm{TeV}$, ATL-PHYS-PUB-2015-035, https://cds.cern.ch/record/2042155.

[14] M. Cacciari, G. P. Salam, G. Soyez, The Catchment Area of Jets, JHEP 0804 (2008) 005, arXiv:0802.1188

[15] ATLAS Collaboration, Expected performance of the ATLAS b-tagging algorithms in Run-2, ATL-PHYS-PUB-2015-022, https://cds.cern.ch/record/2037697

[16] ATLAS Collaboration, Search for diboson resonances with boson-tagged jets in pp collisions at $\sqrt{s}=13 \mathrm{TeV}$ with the ATLAS detector, arXiv:1708.04445v1 [hep-ex].

[17] ATLAS Collaboration, Search for heavy resonances decaying to a $W$ or $Z$ boson and a Higgs boson in the $q \bar{q}\left({ }^{\prime}\right) b \bar{b}$ final state in pp collisions at $\sqrt{s}=13 \mathrm{TeV}$ with the ATLAS detector, arXiv:1707.06958v1 [hep-ex].

[18] ATLAS Collaboration, Search for pair production of Higgs bosons in the $b \bar{b} b \bar{b}$ final state using proton-proton collisions at $\sqrt{s}=13 \mathrm{TeV}$ with the ATLAS detector, ATLAS-CONF-2016-049, http://cds.cern.ch/record/2206131.

[19] ATLAS Collaboration, Searches for heavy ZZ and ZW resonances in the llqq and vvqq final states in pp collisions at $\sqrt{s}=13 \mathrm{TeV}$ with the ATLAS detector, arXiv:1708.09638v1 [hep-ex].

[20] ATLAS Collaboration, Search for WW/WZ resonance production in lvqq final states in pp collisions at $\sqrt{s}=13 \mathrm{TeV}$ with the ATLAS detector, ATLAS-CONF-2017-051, http://cds.cern.ch/record/2273867. 